\title{
The Application of Mid-Side Theory to Produce Analog Stereo Audio Records Using a Single Laser Beam
}

DOI:

10.17743/jaes.2020.0007

\section{Document Version}

Accepted author manuscript

Link to publication record in Manchester Research Explorer

\section{Citation for published version (APA):}

Wilson, D., Allegre, O., Heinemann, R., Orchid, S., \& Whitehead, D. (2020). The Application of Mid-Side Theory to Produce Analog Stereo Audio Records Using a Single Laser Beam. Journal of the Audio Engineering Society, 68(4). https://doi.org/10.17743/jaes.2020.0007

\section{Published in:}

Journal of the Audio Engineering Society

\section{Citing this paper}

Please note that where the full-text provided on Manchester Research Explorer is the Author Accepted Manuscript or Proof version this may differ from the final Published version. If citing, it is advised that you check and use the publisher's definitive version.

\section{General rights}

Copyright and moral rights for the publications made accessible in the Research Explorer are retained by the authors and/or other copyright owners and it is a condition of accessing publications that users recognise and abide by the legal requirements associated with these rights.

\section{Takedown policy}

If you believe that this document breaches copyright please refer to the University of Manchester's Takedown Procedures [http://man.ac.uk/04Y6Bo] or contact uml.scholarlycommunications@manchester.ac.uk providing relevant details, so we can investigate your claim.

\section{OPEN ACCESS}




\title{
The Application of Mid-Side Theory to Produce Analog Stereo Audio Records Using a Single Laser Beam
}

\author{
D. WILSON', O. ALLEGRE', R. HEINEMANN', S. ORCHID, AND D. WHITEHEAD' \\ (daniel.wilson@manchester.ac.uk) \\ ${ }^{1}$ Department of Mechanical, Aviation and Civil Engineering, School of Engineering, Faculty of Science and \\ Engineering, Manchester University
}

\begin{abstract}
The recent resurgence of vinyl music records sales led by a consumer demand is increasing faster than production capability. This has resulted in supply delays across the sector. Thus far, manufacturing investments have been focused on traditional proven methods rather than alternative technologies. This paper demonstrates for the first time the production of a stereo recording via analog methodology using a single pulsed laser beam. Using mid-side theory, to combine a sum (mono) signal with a difference signal, a $532 \mathrm{~nm} \mathrm{Nd:Yag} \mathrm{laser} \mathrm{beam} \mathrm{was}$ used to process high-impact polystyrene discs (HIPS). Stereo recordings were manufactured by varying the laser power to produce a difference signal and deflecting the beam with a mirror mounted galvanometer to produce the sum signal. Upon playback on a conventional turntable, the recordings were analyzed with an oscilloscope and stereo separation was observed. To our knowledge this is the first time a stereo signal has been successfully recorded using a single laser beam. Previous literature has used a single laser beam to achieve mono signals and required significant digital pre-processing of the audio source. This new methodology requires lower investment costs than traditional pressing plants and would make volume-tailored production more affordable.
\end{abstract}

\section{INTRODUCTION}

Traditional vinyl record production methods are focused on mass production and have changed very little since Blumlein's patent in 1931 [1]. Recently, there has been a strong resurgence for music in this format. This increase in demand has outpaced production investments, resulting in a severe lack of manufacturing capacity. As this resurgence is only recent, there has been limited research into alternative production methods. There are two obvious avenues of research to apply new manufacturing technologies: either creating a new way to manufacture a record or adapting the existing processes to increase production. For example developing new methods to make stampers for pressing plants, thereby eliminating bottlenecks associated with cutting lacquers and electroforming negatives. Thus far modern manufacturing methods such as 3D printing and laser etching have been used to produce mono recordings on discs.

In the 1960s, when mono recordings were superseded by stereo, a key requirement was that the associated technology was backwards compatible. A mono record has the single channel of audio engraved on the disc in the form of a horizontal modulation of the groove across the disc surface. As a result a mono record groove has a constant width and depth. A stereo record is produced by changing the width and depth of the groove. The horizontal groove modulation carries the sum (mono) of the signal information for the two channels. The vertical modulation of the groove imparts the difference signal of the two channels on the stylus. Therefore, recordings with large groove width changes have a greater stereo image. Fig. 1 illustrates the sum and difference movements of a stylus on a stereo record.

Vinyl is used to make records because it is easy to press. As it is a soft material it suffers from degradation of quality after excessive playback, manhandling, or poor storage. The use of a laser opens up the possibility of utilizing materials with more favorable characteristics. Using lasers for microprocessing is an extensive research area and has covered the production of microstructures in polymers to the scale necessary for records [2-8]. The resurgence of vinyl record production means that laser technology is becoming an alternative to the traditional production methods. Lasers have several advantages over using a cutting stylus; they do not blunt and do not create any resistance on the rotating disc. They can be coupled to CNC systems to 


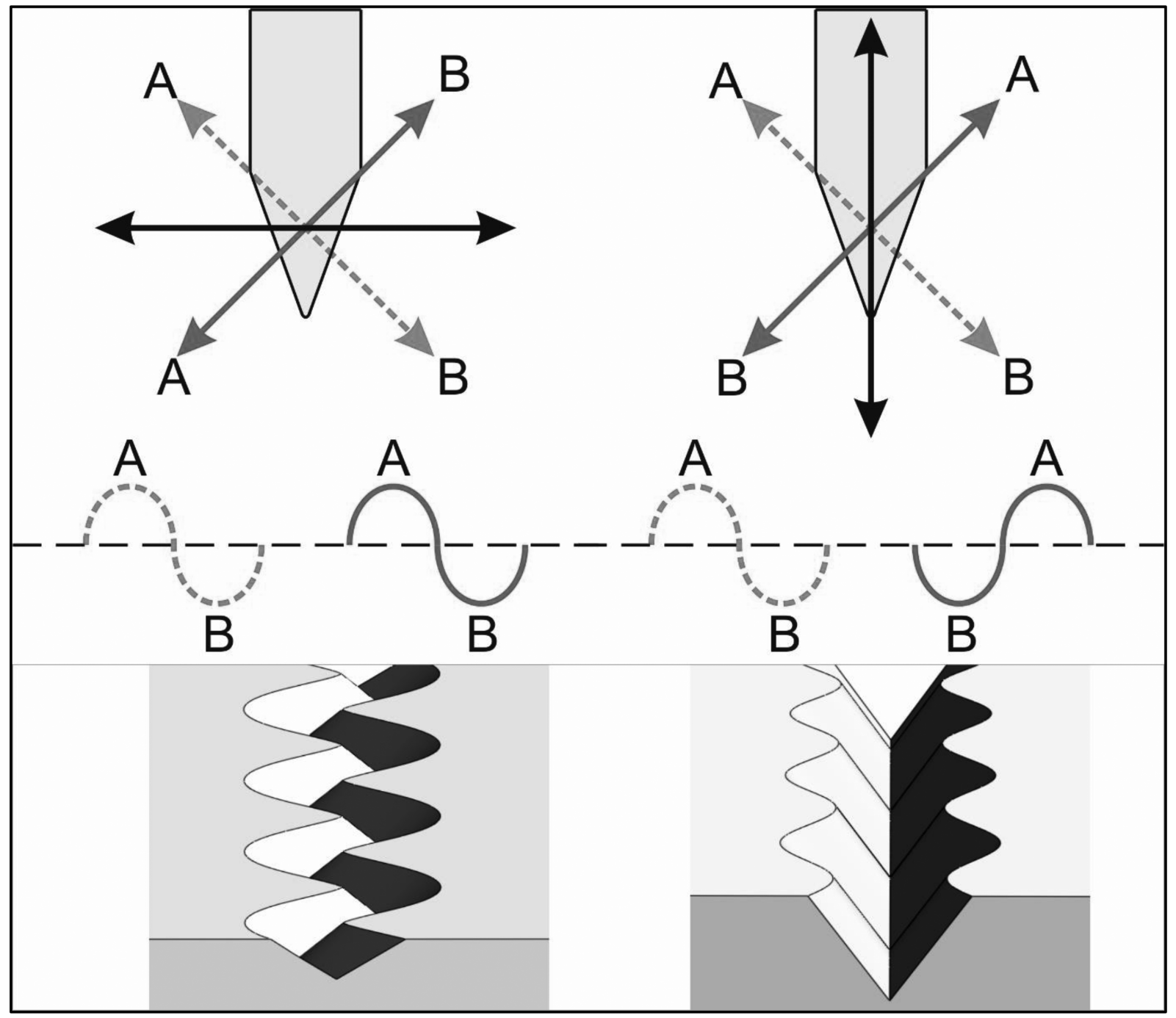

Fig. 1. Schematic representation of stylus displacement when a record is played, illustrating the playback movements of the stylus as sum (left illustrations) and difference (right) signals are applied. The black arrowed line shows the overall movement of the stylus when subjected to the two signals. The illustrated parts in dashed represent the left channel and the continuous represents the right channel. At the bottom of the figure are illustrations representing the record grooves. Note that all of the stylus movements are in-phase.

provide disc rotation and a transverse feed to produce the necessary spiral. There are a wide range of optical methods available to manipulate beams at high speed [9-12]; this means that potentially the data could be transcribed faster than real time.

Ghassaei $[13,14]$ has used stereo source material to produce low-resolution mono recordings. The soundwave was digitized to produce a $3 \mathrm{D}$ representation of a groove with $\mathrm{X}$, $\mathrm{Y}$, and $\mathrm{Z}$ co-ordinates at each sample point. This data was optimized for either 3D printing or laser engraving. The research using a 3D printer [13] modulated the groove vertically, producing a difference signal, rather than a mono signal. Further work by Ghassaei [14] focused on using a laser to produce horizontally modulated mono recordings on wood. The surface roughness of the disc material added further distortion to the source material. This research produced very low resolution recordings. Both the 3D printer and laser engraver used only had capability to store small data files and were not capable of sufficient axis resolution.
A recent patent by Loibl and Schmidt [15] details the digitization of the soundwaves to produce a stereo record using a similar methodology to Ghassaei [13,14]. A complex twin laser beam system is used to replicate a similar cutting process to that detailed by Blumlein [1]. A second methodology describes engraving the disc using a single laser directed at a mirror mounted galvanometer system. A possible complication with this method is that the laser beam interacts with the surface at an angle rather than perpendicularly. This produces feature errors. This methodology requires removal of material using multiple passes of the laser. This is fairly complex as the digital file size is very large and the galvanometer system may not be able to exactly overlay each pass. The authors also propose that they can manufacture stampers for pressing plants using these methodologies. The patent covers methods that are unproven.

Here we demonstrate proof of concept of a new methodology that can be used to produce a stereo recording using a laser. Mid-side processing is a studio tool used to 


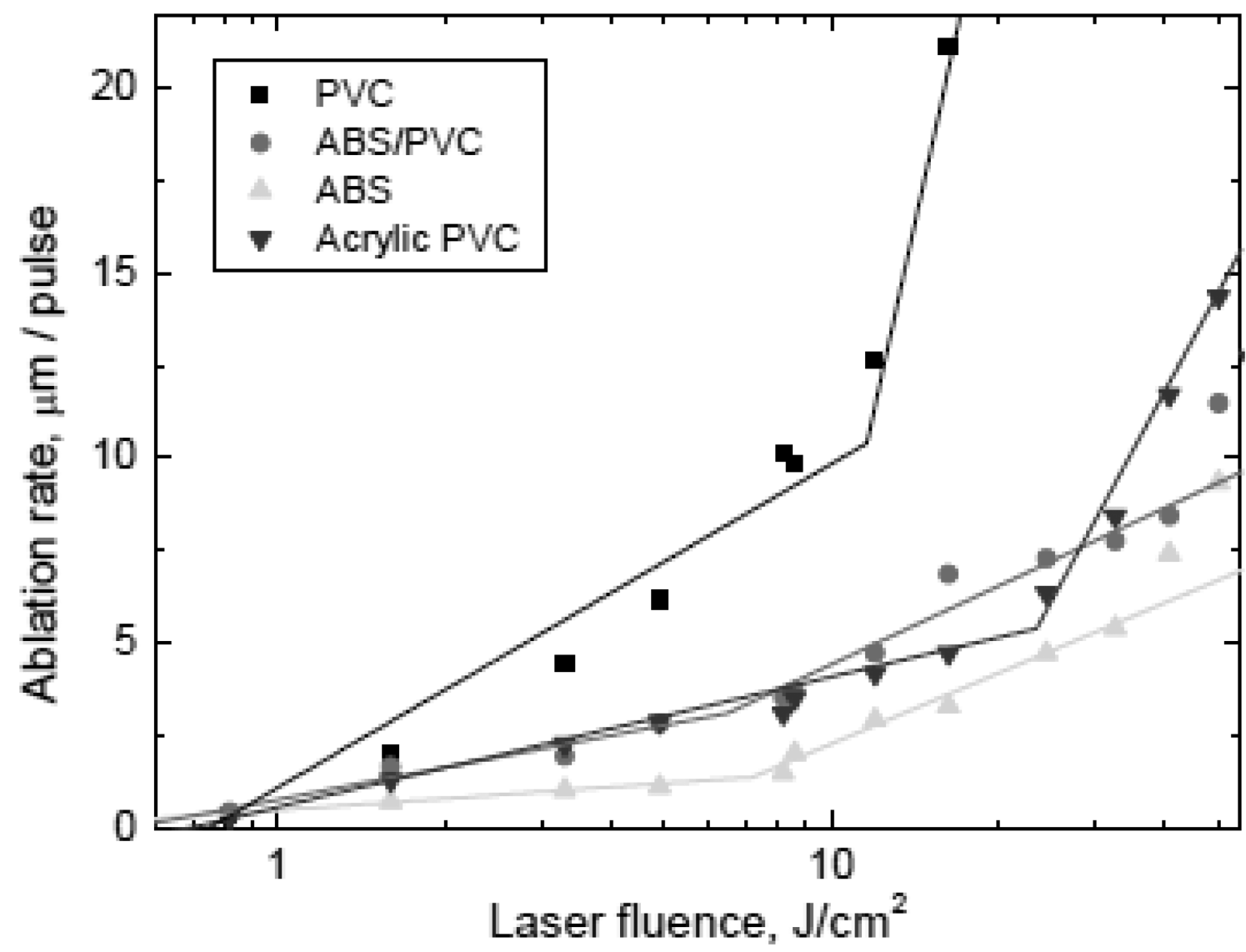

Fig. 2. An example of ablation rate against fluence, using different polymers exposed to a $266 \mathrm{~nm}$ UV wavelength laser, with $60 \mathrm{ps}$ pulse length [8].

manipulate the stereo field [16]; here we apply it to the manufacture of records using a pulsed laser. It is used to rotate stereo information so that machining is in the vertical and horizontal axis. This eliminates the need to cut at $45^{\circ}$ to the surface for each channel as is the norm. A laser can be used to produce the difference information (vertical) by varying the laser intensity. Horizontal manipulation of the laser beam produces the sum information. The source material can be processed using simple ana$\log$ circuitry without any digitization and can be used to directly control the processing equipment. This simplifies the process and retains the tradition of the format. An advantage of using this technology would be that it requires less investment than a conventional pressing plant and lower running costs, which makes it more economically viable to produce small runs.

\section{PRINCIPLES OF THE METHOD}

The groove width of a typical record is $50.8 \mu \mathrm{m}$; a laser is easily capable of producing a feature in this range [2-8] as the minimum spot size of a Gaussian laser at its focal position is typically twice its wavelength (Khan-Malek, 2006) [4]. Therefore, using a shorter wavelength will produce a narrower groove. Theoretical focal diameters can be calculated using the following simplified formula. This does not account for beam divergence or the $\mathrm{M}^{2}$ factor (beam quality).

$$
W_{f}=\frac{\lambda f}{\pi W_{O}}
$$

Where $W_{f}$ is the focal diameter, $f$ the focal length of lens, and $W_{O}$ the beam diameter before the lens.

As a record groove is engraved in a spiral toward the center, the linear speed decreases from the outer edge. Using the RIAA standards this equates to $504 \mathrm{~mm} \mathrm{~s}^{-1}$ at the outer edge, reducing to $208 \mathrm{~mm} \mathrm{~s}^{-1}$ at the inner diameter. This means there is an increase in laser interaction time resulting in an increasing groove size. Therefore, the laser power will have to be reduced as the laser engraves toward the center to compensate. Reducing the repetition rate of a pulsed laser will also have the effect of reducing the laser power, provided that pulse energy remains constant. Decreasing repetition rate will reduce pulse overlap as the recording progresses toward the center. There are many studies on pulse overlap on ablation rates and efficiencies $[17,18]$. Obviously the repetition rate has to be sufficient so that it will not affect the audio recording.

Varying laser power has a direct effect on the rate of material removal. Laser ablation rates have some form of linear response as the energy, or fluence, of the laser is increased [8,19-24]. However, the linear response may be limited. In 


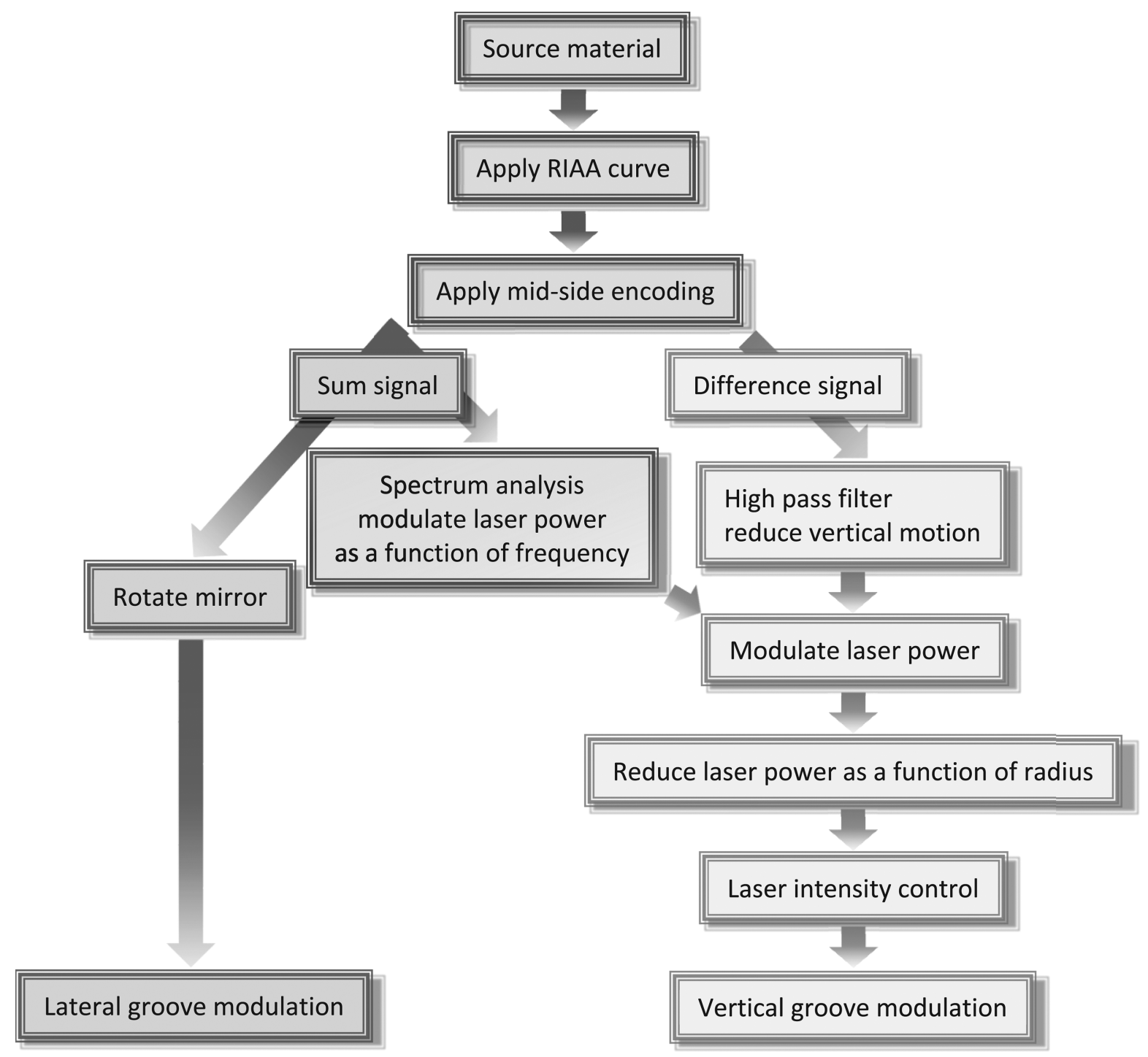

Fig. 3. Processes to manufacture a stereo record.

a Gaussian beam, as the intensity increases the effective diameter of the beam increases. This is due to the threshold fluence of the material being exceeded at increased distance from the center of the beam as the intensity rises. Therefore groove depth can be controlled by varying laser intensity.

Care has to be taken to select a material that has the necessary rate of removal and matches the wavelength and pulse duration of the laser. There are many polymers and metals that have an excellent linear response [8,19-22]. Fig. 2 illustrates the relationship of fluence against ablation for polymers irradiated with UV laser pulses [8]. There are normally two distinct phases of useful ablation. The first is used for surface modification, such as polishing, or photo degradation, associated with thermal damage of the polymer. Then there is a second linear response phase that is from the photochemical breaking of bonds. Lastly there is an area of processing that is characterized by excessive thermal damage and the possible generation of plasma. When selecting materials a balance needs to be sought between ablation rate and suitability of the material for the final product. High-impact polystyrene has been used in the past to make 7" singles; it has a good process window in similar experimental conditions [23] to those detailed in this paper.

Converting a sound recording into a stereo record using mid-side processing involves several processes. Fig. 3 details these steps. Firstly the RIAA frequency curve has to be applied to the audio. Then apply the mid-side encoding. As the sum frequency increases the laser will be translated across the surface at a greater speed. This will reduce the depth of the groove; therefore a weighting will have to be applied to the difference signal to keep the groove depth constant. The sum signal can then be input directly into a rotational mirror with a little correction to account for the frequency response of the mirror. After the mid-side encoding the difference signal will need to have a high pass filter applied to restrain the needle from jumping out of the 
groove. Then the weighting factor from the sum signal is applied. A further weighting will need to be applied to the DC difference signal to account for the rotational velocity decrease as the engraving progresses toward the center of the disc; otherwise the groove will increase in depth and width.

\section{EXPERIMENTAL DETAILS}

The laser used for this investigation was a Spectra Physics Navigator II Nd:YAG with a 532-nm harmonic head. A repetition rate of $20 \mathrm{KHz}$ was used as this emitted a more stable beam with a slightly improved linear power output to analog input. The analog input of this laser accepts a DC $0-6 \mathrm{~V}$ signal; this directly controls the current in the charge amplifier of the laser. The pulse length was $35 \mathrm{~ns}$, and a beam expander was utilized to increase the beam diameter to $10 \mathrm{~mm}$. Once focused through a flat field $50 \mathrm{~mm}$ focal length lens, the laser focal diameter was calculated at 55 um. A cast high-impact polystyrene (HIPS) disc was placed in the focal plane of the lens and rotated using an Aerotech CNC system. Fig. 4 illustrates the experimental setup.

The maximum frequency of the difference signal was limited by the frequency response of the analog input. The maximum analog input frequency was $10 \mathrm{~Hz}$. As this frequency was below the sensitivity of the playback stylus the rotational speed of the laser processing was decreased to $1.65 \mathrm{rpm}$ rather than the standard $33 \mathrm{rpm}$. This resulted in a full rotation taking 36 seconds rather than $1.82 \mathrm{sec}-$ onds, as in the case of a conventional $33 \mathrm{rpm}$ recording. The repetition rate of the laser was set to $20 \mathrm{KHz}$, which during $33 \mathrm{rpm}$ playback would be the equivalent of 400 $\mathrm{KHz}$. This would result in the analog input frequency being pitch shifted to $200 \mathrm{~Hz}$ upon playback. A signal generator was directly coupled to the laser analog input, with a $1.6-\mathrm{V}$ voltage offset to produce a groove. Peak-to-peak voltage was calibrated to produce a variance in power sufficient to create a measurable change in the groove cross section on the disc; this was $50 \mathrm{mV}$. A groove without modulation was produced at $200 \mathrm{~mW} \pm 10 \mathrm{~mW}$. When applying vertical modulation an oscillating signal with a peak-to-peak power of $50 \mathrm{~mW} \pm 10 \mathrm{~m} \mathrm{~W}$ produced a groove with a minimum power of $150 \mathrm{~mW}$ and maximum of $250 \mathrm{~mW}$. This equates to a fluence of $0.84 \mathrm{~J} \mathrm{~cm}^{2}$, which is similar to investigations by other researchers using this material $[22,23]$. The errors for measuring the power output of the laser are associated with power fluctuations from thermal cycling of the cooling system.

Horizontal modulation of the groove was controlled using a mirror mounted on a conventional analog galvanometer driven by an audio amplifier. The $25-\mathrm{Hz}$ sine wave was produced using a signal generator. After amplification this had an amplitude of $40 \mathrm{mV}$ on the galvanometer coil, which provided sufficient displacement of the mirror. Upon playback this would result in a $500-\mathrm{Hz}$ signal.

Once the grooves were produced the disc was transferred to a conventional record turntable. During playback the stylus was able to convert the groove information into a stereo signal as described in Fig. 1. The recording was captured and saved as a WAV using Audacity. A PicoScope digital PC oscilloscope was used to analyze the waveforms. A Keyence digital microscope and NanoFocus laser profilometer were used to analyze the groove geometries.

\section{RESULTS}

A cross-sectional profile of a record groove inscribed in HIPS, using the described methodology, is shown in Fig. 5. The groove has sloped sides with a wide base rather than the "vee" seen in conventional records. It can also be seen that the side walls rise above the surface of the disc to form horns. Without modulation the groove width was $125 \mu \mathrm{m} \pm 2 \mu \mathrm{m}$ with a depth of $60 \mu \mathrm{m} \pm 2 \mu \mathrm{m}$. Applying an oscillating voltage to the laser produced a peak-to-peak modulated groove width and depth of $60 \mu \mathrm{m} \pm 2 \mu \mathrm{m}$. The horizontal modulation was $200 \mu \mathrm{m} \pm 2 \mu \mathrm{m}$ peak-to-peak and is imaged in Fig. 6. In a conventional microgroove record the groove width is $25-100 \mu \mathrm{m}$ with a maximum horizontal peak-to-peak modulation of $50 \mu \mathrm{m}$ and a depth of $25-125 \mu \mathrm{m}$.

Oscilloscope traces of the various grooves produced are shown in Fig. 7. Graphs A-C show distortion; this is explained in the discussion. Frequencies below $125 \mathrm{~Hz}$ and above $800 \mathrm{~Hz}$ were removed using software low and high pass filters in Adobe Soundbooth (Graph D). This minimized the effect of the distortion, thereby allowing comparison to the theoretical soundwave (Graph E). Graph E is the theoretical signal calculated using the mid-side formulas. A 2-ms delay was added to the difference signal (vertical) of Plot E as the signal generators were not synchronized.

\section{DISCUSSION}

Initial tests using polyvinyl chloride (PVC) as the disc material revealed that the recording was unplayable due to excessive oxidation and vulcanization of the polymer. PVC is an unfavorable material to work with when laser processing as it breaks down to release chloride gasses and free radicals [25]. Therefore regardless of the result observed from PVC an alternative material was always going to be a necessity.

Several other polymers were investigated, with highimpact polystyrene (HIPS) producing the best result. With HIPS the grooves were seen to exhibit noise, as shown in Fig. 7(a)-7(c). The predominant reason for this was the choice of material was not optimal for the laser system. Further research is ongoing to address this. Other factors that contributed to the degraded signal are from unwanted mechanical vibrations of the equipment and mains hum.

Surface noise and groove deformation were a result of thermal degradation of the HIPS and debris from the machining process. This is due to photothermal machining occurring, whereby the photons in the beam have insufficient energy to break the polymers. Instead the photons excite the bonds of the polymer, resulting in a material that ablates by boiling [3-6]. To prevent photothermal machining a shorter wavelength can be used, which should improve the groove precision. UV lasers emit photons with a high 


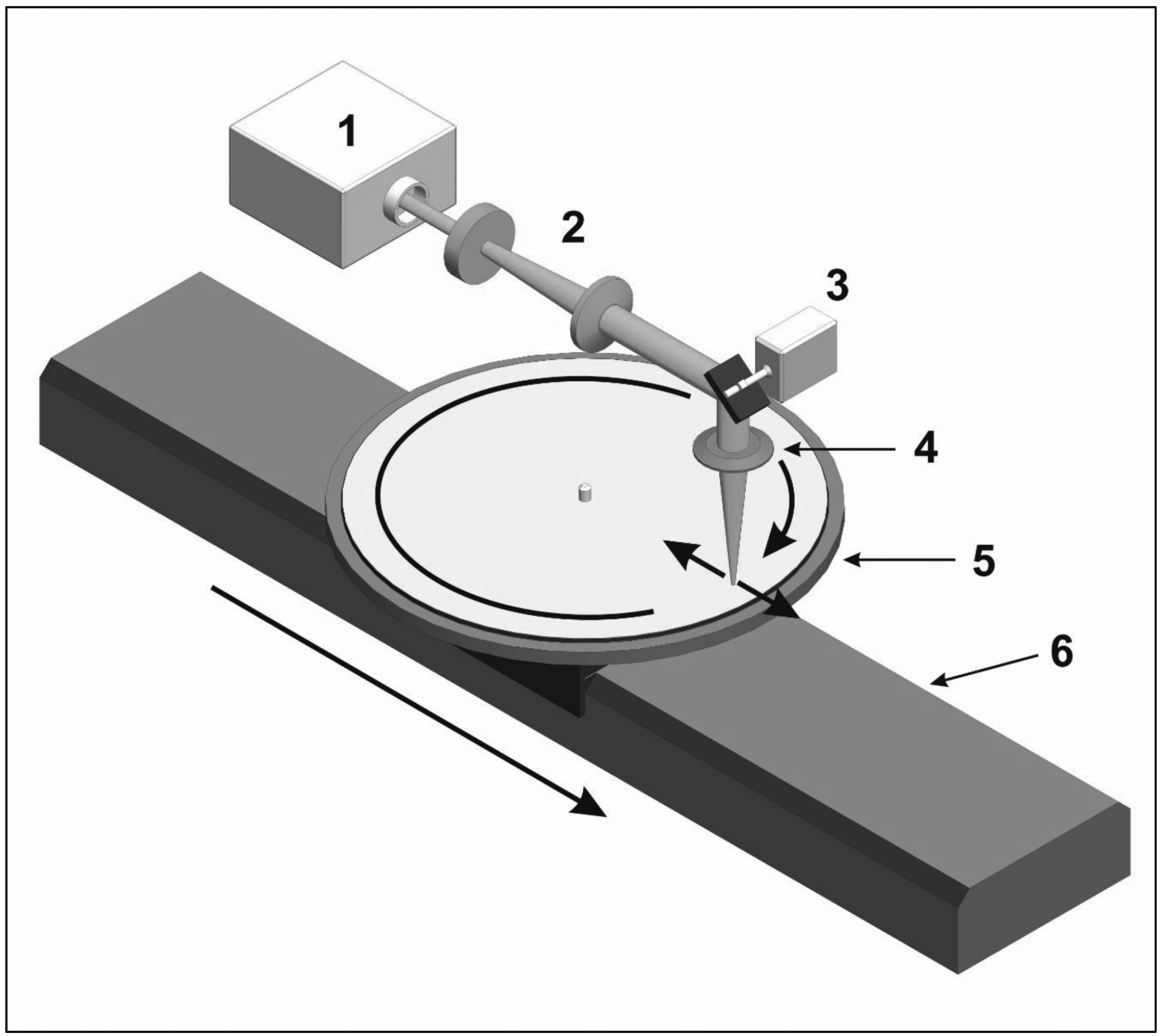

Fig. 4. Experimental set-up. Annotations: 1: laser source. 2: Galileo beam expander. 3: rotating galvanometer mirror. 4: 50-mm focal lens. 5: rotating platter. 6: CNC stage for horizontal motion.

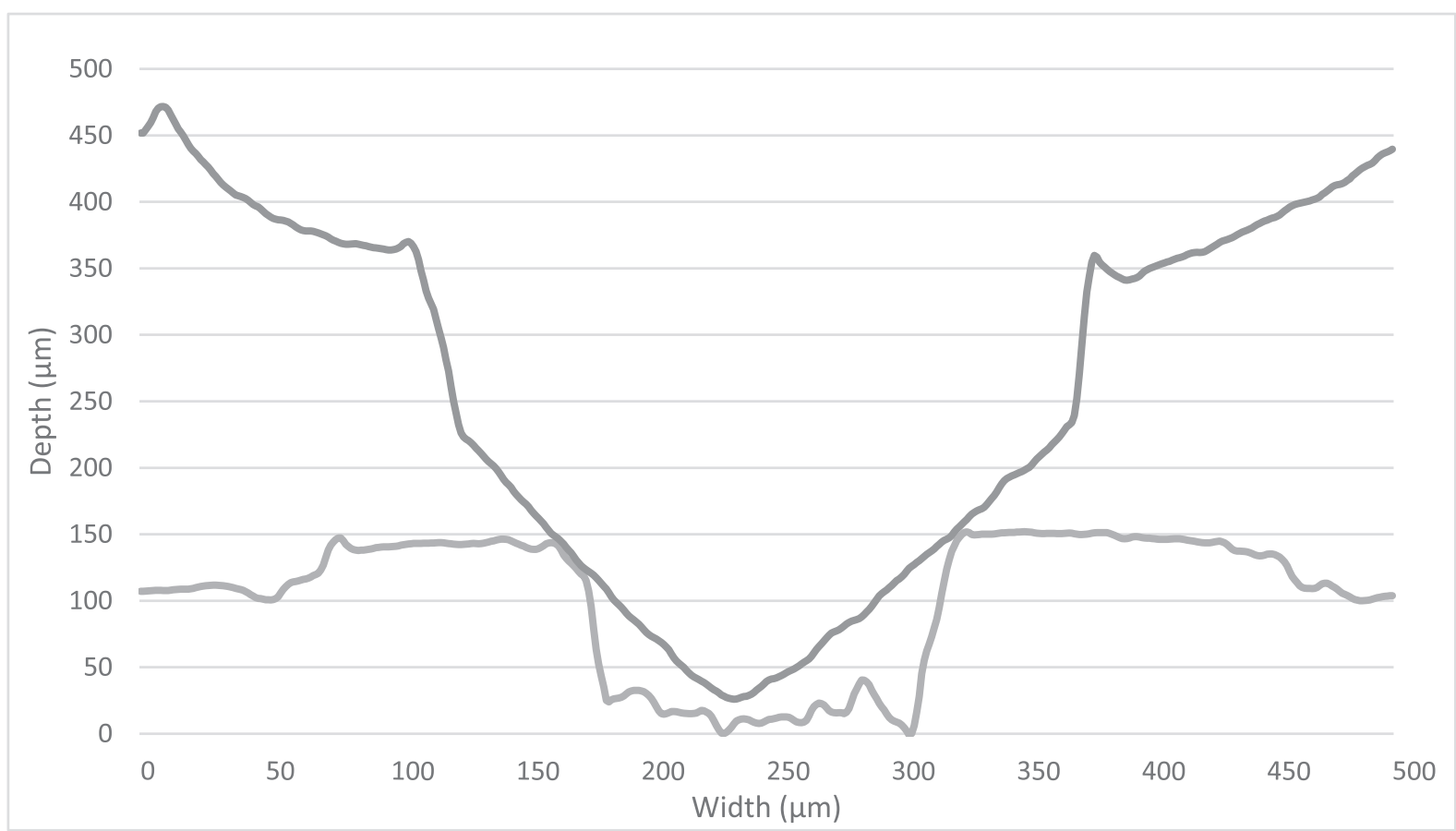

Fig. 5. Scanned HIPS groove with a scanned stylus overlaid. Scanned using a NanoFocus $\mu$ Scan laser profilometer. 


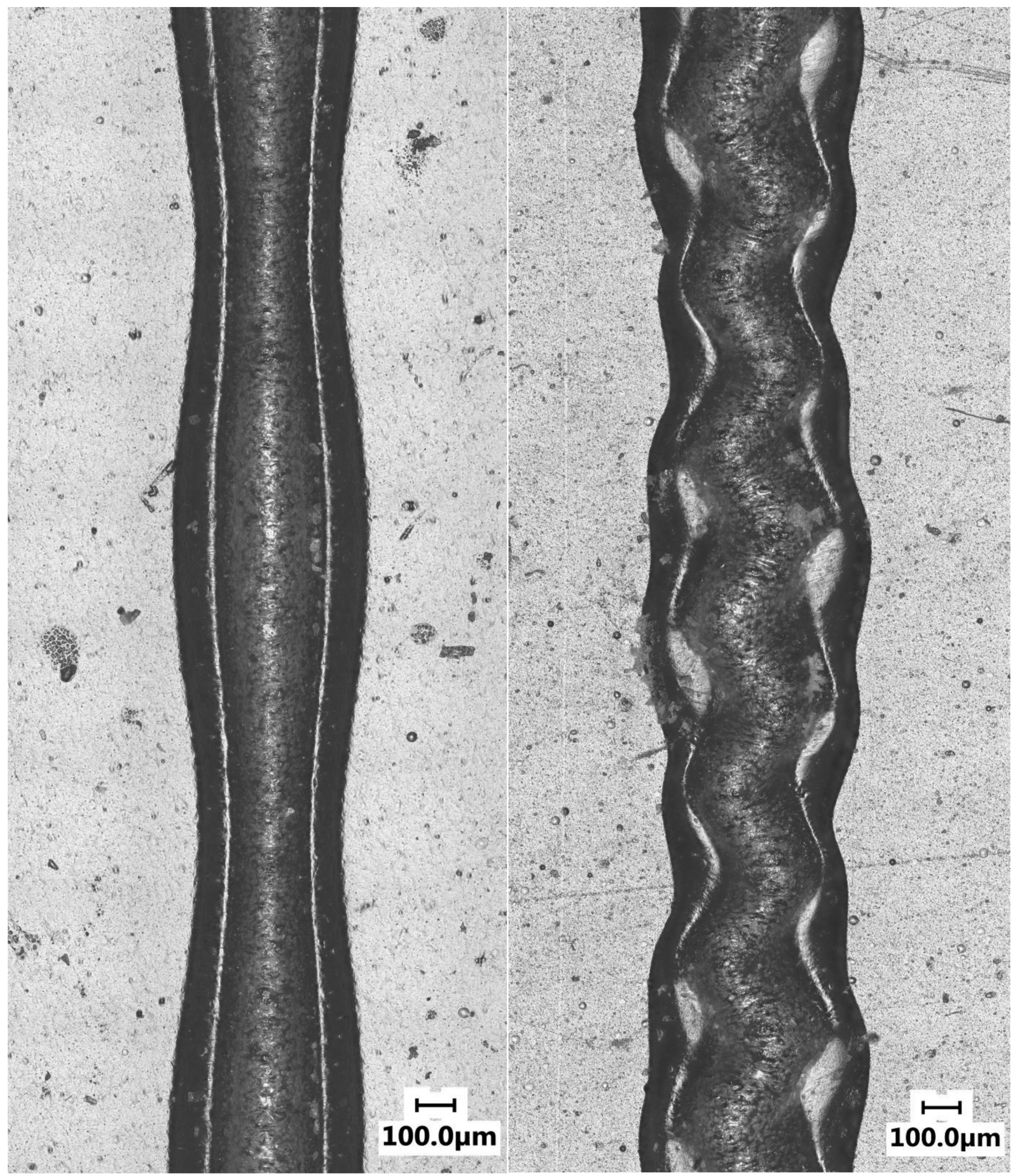

Fig. 6. Microscope images of modulated groove: difference signal produced by modulating laser intensity at $10 \mathrm{~Hz}$ to produce vertical modulation (left); stereo modulation $25 \mathrm{~Hz}$ lateral and $10 \mathrm{~Hz}$ vertical (right).

energy, such as $355 \mathrm{~nm}$. The high-energy photons promote photochemical interactions, leading to clean surface ablation by breaking the chemical bonds $[3,4]$. This results in a superior surface finish with less charring or vulcanization of polymers [5,6]. The use of ultrashort pulse length, typically a picosecond or less, can also improve the surface finish of processed polymers [7], the theory being that the pulses are of a sufficiently short period such that there is insufficient time for the energy to propagate through the material. Thermal energy is transferred to the ejected material rather than being absorbed into the bulk $[7,8]$.

Fig. 5 shows the etched groove is more of a " $U$ " rather than a "vee" profile. This is due to the beam interaction with the polymer and the relatively long depth of field of the focal lens used. The shape of the beam is Gaussian and therefore produces a " $U$ " shape due to its energy distribution 

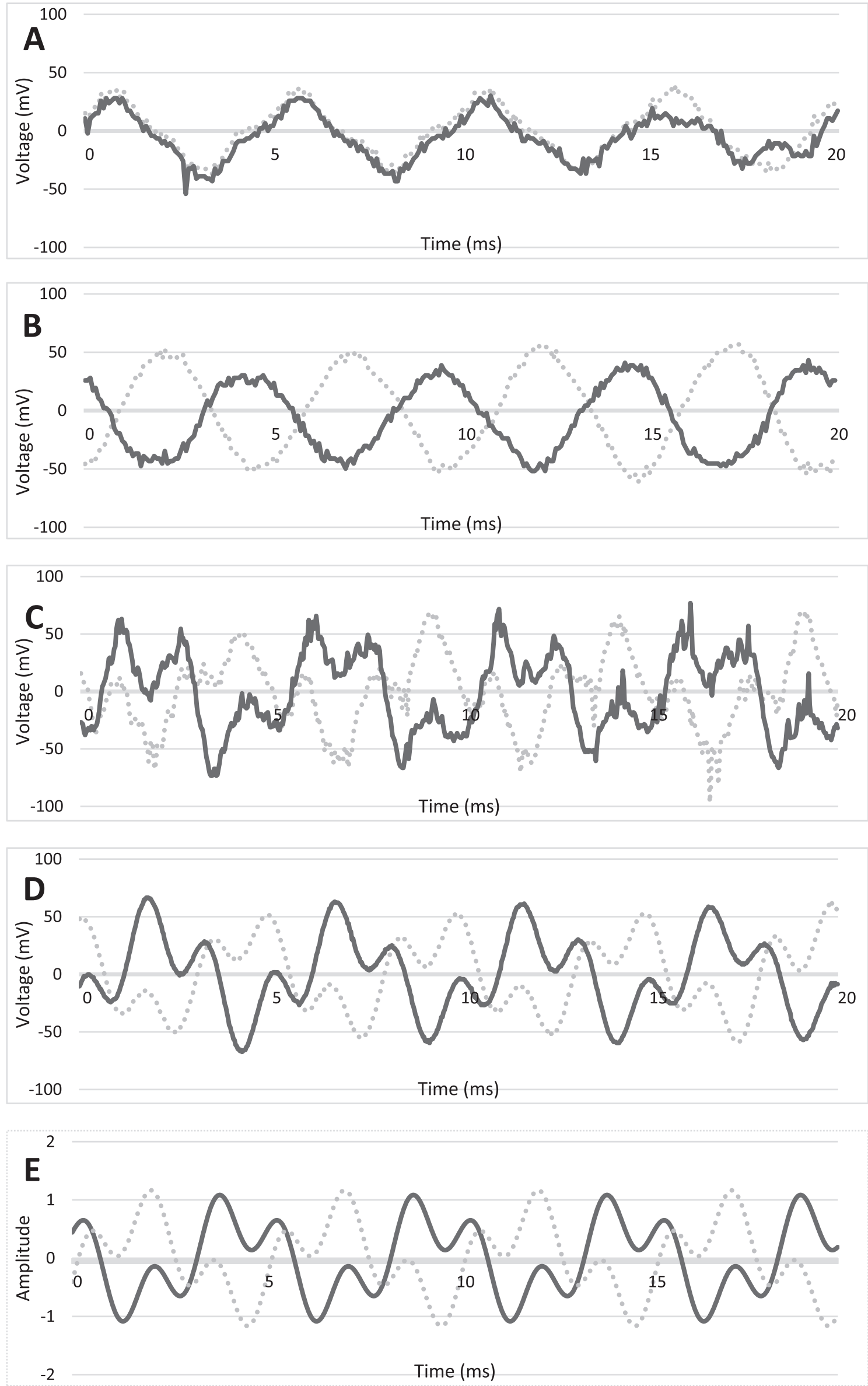

Fig. 7. Record signal plots in descending order; (a) sum, (b) difference, (c) stereo raw signal, (d) stereo with noise reduced, and (e) theoretical signal. Continuous and dotted traces are the signals picked up on the left and right channels of the stylus. 
profile. Fig. 5 shows a HIPS groove overlaid with a stylus. The stylus can be seen to be resting on the horns of the groove. This groove geometry results in a reduced contact area when compared to a traditionally cut record, which would wear quicker. Future investigations are planned to combine the methodology detailed here with beam shaping. Laser beams can be shaped into different energy profiles, allowing the beam to be engineered into a more preferable distribution. Depending on the stylus contact area, this may mean that focusing on achieving a groove that eliminates base roughness is unnecessary. There are a variety of beam shaping techniques which includes masking the beam and using a spatial light modulator $[20,26,28]$.

The vertical component of the groove was controlled by varying the laser power. The maximum power modulation frequency of the laser was found to be limited to $10 \mathrm{~Hz}$. This limits the speed of the recording to a maximum of $1 / 20^{\text {th }}$ of a $33 \mathrm{rpm}$. A possible solution is to use PWM frequency control of the laser to modulate the laser power. However, this will produce bursts of pulses that may produce groove distortion. Further research is investigating other methods to vary laser power with a similar bandwidth to conventional records.

As lateral groove modulation is controlled using a mirror mounted on an analog galvanometer, the available speed is limited. Higher frequency response and precision galvanometers are available. A mono recording was used to analyze the galvanometer response to a sinewave. Plot A of Fig. 7 shows that the soundwave captured is more of a triangle rather than a pure sinewave. This can be attributed to the non-linear response of the galvanometer. The evidence of this distortion is also seen in Plot $\mathrm{C}$, where the peaks are fairly angular. This is not unexpected given the age of the galvanometer available at the time this research was carried out. This is expected to be minimized with the installation of a new galvanometer. In recent years galvanometer technology has been somewhat superseded by polygon scanners. A polygon scanner can achieve scan rates exceeding $250 \mathrm{~m}$ $\mathrm{s}^{-1}$ over ten times the rate of a conventional scanner [26].

\section{CONCLUSION}

The results show that a stereo record groove can be produced from a single laser beam with a minimum of signal processing. Mid-side audio processing enables the limiting degrees of freedom of a laser system to be used to create a stereo recording that reproduces that of traditionally engraved records. These initial investigations have identified the key areas that require further research and refinement. Research into alternative production methods for records have thus far been limited. This research opens up a new avenue of investigation.

\section{REFERENCES}

[1] A. D. Blumlein, "Improvements in and Relating to Sound-Transmission, Sound-Recording and Sound-Reproducing Systems," British Patent Specification 394,325 (1931).
[2] S. Ravi-Kumar, B. Lies, X. Zhang, H. Lyu and H. Qin, "Laser Ablation of Polymers: A Review," Polym. Int., vol. 68, no. 8 (2019), https://doi.org/10.1002/ pi.5834.

[3] P. E. Dyer, "Excimer Laser Polymer Ablation: Twenty Years on," Appl. Physics A, no. 77, pp. 167-173 (2003), https://doi.org/10.1007/s00339-003-2137-1.

[4] C. G. Khan-Malek, "Laser Processing for Biomicorfluidics Applications (Part I)," Analyt. Bioanalyt. Chem., vol. 385, no. 8, pp. 1351-1361 (2006), https://doi.org/10.1007/s00216-006-0514-2.

[5] V. Srinivasan, M. A. Smrtic and S. V. Babu, "Excimer Laser Etching of Polymers," J. Appl. Phys., vol. 59, no. 11, pp. 3861-3867 (1986), https://doi.org/10.1063/ 1.336728 .

[6] C. A. Aguilar, Y. Lu, S. Mao and S. Chen, "Direct Micro-patterning of Biodegradable Polymers Using Ultraviolet and Femtosecond Lasers," Biomaterials, no. 26, pp. 7642-7649 (2005), https://doi.org/ 10.1016/j.biomaterials.2005.04.053.

[7] J. Sercel, J. Bickley and J. Stearns, "Ultrafast Lasers: Picosecond Laser Micromachining Produces High Quality Results," https://www.laserfocusworld.com/ articles/print/volume-48/issue-10/features/ultrafast-laserspicosecond-laser-micromachining-produces-high-qualityresults.html, accessed: 2018-05-23.

[8] G. Raciukaitis and M. Gedvilas, "Processing of Polymers by UV Picosecond Lasers," presented at the Proceedings of the 24th ICALEO Conference (2005), http:// www.baltfab.com/wp-content/uploads/2014/03/processingof-polymers-by-uv-picosecond-lasers.pdf.

[9] M. Malinauskas, A. Žukauskas, S. Hasegawa, YV. Mizeikis . Hayasaki, R. Buividas and S. Juodkazis, "Ultrafast Laser Processing of Materials: From Science to Industry," Light Sci. Appl. (2016), https://doi.org/10.1038/ lsa.2016.133.

[10] I. Gnilitskyi, T. J. -Y. Derrien, Y. Levy, N. M. Bulgakova, T. Mocek and L. Orazi, "Highspeed Manufacturing of Highly Regular Femtosecond Laserinduced Periodic Surface Structures: Physical Origin of Regularity," Sci. Rep. (2017), https:// doi.org/10.1038/s41598-017-08788-z.

[11] J. Schille, L. Schneider, A. Streek, S. Kloetzer and U. Loeschner, "High-Throughput Machining Using a High-Average Power Ultrashort Pulse Laser and High Speed Polygon Scanner," Opt. Eng. (2016), https://doi.org/10.1117/1.OE.55.9.096109.

[12] Y. Jin, O. J. Allegre, W. Perrie, K. Abrams, J. Ouyang, E. Fearon, S. P. Edwardson and S. P. Dearsden, "Dynamic Modulation of Spatially Structured Polarization Fields for Real-Time Control of Ultrafast LaserMaterial Interactions," Optics Expr., vol. 21, no. 21 (2013), https://doi.org/10.1364/OE.21.025333.

[13] A. Ghassaei, "3D Printed Record," http:// www.amandaghassaei.com/projects/3D_printed_record/, accessed: 2018-05-10.

[14] A. Ghassaei, "Laser Cut Record," http:// www.amandaghassaei.com/projects/laser_cut_record/, accessed: 2018-05-10. 
[15] G. Loibl and V. Schmidt, "Method for Producing a High Definition Analogue Audio Storage Medium," US Patent US20190065662A1 (2019).

[16] W. L. Dooley and R. D. Streicher, "M-S Stereo: A Powerful Technique for Working in Stereo," J. Audio Eng. Soc., vol. 30, no. 10, pp. 701-718 (1982), www.aes.org/elib/browse.cfm?elib=3814.

[17] K Elizabeth. E. K. Illy, D. J. W. Brown, M. J. Withford and J. A. Piper, "Optimization of Trepanning Strategies for Micromachining of Polymers With High-PulseRate UV Lasers," High-Power Lasers Manuf., vol. 3888 (2000), https://doi.org/10.1117/12.377071.

[18] L. E. Criales, P. F. Orozco, A. Medrano, C. A. Rodríguez and T. Özel, "Effect of Fluence and Pulse Overlapping on Fabrication of Microchannels in PMMA/PDMS Via UV Laser Micromachining: Modeling and Experimentation," Mater. Manuf. Process., pp. 890-901 (2014), https://doi.org/10.1080/10426914.2015.1004690.

[19] W. Pfleging, M. Przybylski and H. J. Brückner, "Excimer Laser Processing - State of the Art and New Approaches to Microsystem Technology," presented at the Proceedings of SPIE - The International Society for Optical Engineering (2006), https://doi.org/10.1117/ 12.647425 .

[20] T. Lippert, "Interactions of Photons With Polymers: From Surface Modification to Ablation," Plasma Process. Polym., vol. 2, no. 7, pp. 525-546 (2005), https://doi.org/10.1002/ppap.200500036.

[21] A. Bharatish and S. Soundarapandian, "Influence of Femtosecond Laser Parameters and Environment on Surface Textures Characteristics of Metals and Non-metals State of the Art," Lasers Manuf. Mater. Process., vol. 5, no. 2, pp. 143-167 (2018), https://doi.org/10.1007/s40516018-0059-1.

[22] K. Dholakia and T. Čižmár, "Shaping the Future of Manipulation," Nature Phot. (2011), https://doi.org/10.1038/nphoton.2011.80.

[23] S. Arif and W. Kautek, "Pulse Laser Machining and Particulate Separation From High Impact Polystyrene," Appl. Surf. Sci., vol. 288, pp. 9-14 (2014), https://doi.org/10.1016/j.apsusc.2013.08.060.

[24] R. S. Kappes, F. Schönfeld, C. Li, A. A. Golriz, M. Nagal, T. Lippert, H. Butt and J. S. Guttman, "A Study of Photothermal Laser Ablation of Various Polymers on Microsecond Timescales," SpringerPlus (2014), https://doi.org/10.1186/2193-1801-3-489.

[25] E. Stauffer, J. A. Dolan and R. Newman, "Review of Basic Organic Chemistry," in Fire Debris Analysis, pp. 49-83 (2008).

[26] Cambridge Technology, "Polygon Laser Scanner," https://www.cambridgetechnology.com/products/polygonlaser-scanner, accessed: 2019-11-16.

[27] R. J. Beck, A. J. Waddie, J. P. Parry, J. D. Shephard, D. P. Taghizadeh, and D. P. Hand, "Adaptive Laser Beam Shaping for Laser Marking Using Spatial Light Modulator and Modified Iterative Fourier Transform Algorithm," Phys. Proced., vol. 12, pp. 465-469 (2011), https://doi.org/10.1016/j.phpro.2011.03.158.

[28] O. J. Allegre, Z. Li and L. Li, "Tailored Laser Vector Fields for High-precision Micro-manufacturing," CIRP Annals, vol. 68, no. 1, pp. 193-196 (2019), https://doi.org/10.1016/j.cirp.2019.04.125. 


\section{THE AUTHORS}

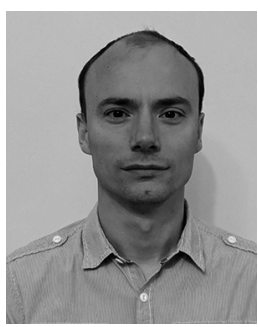

Daniel Wilson

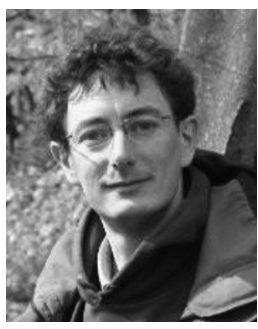

Dr. Allegre

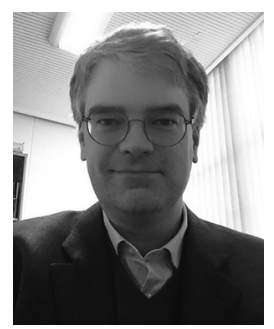

Robert Heinemann

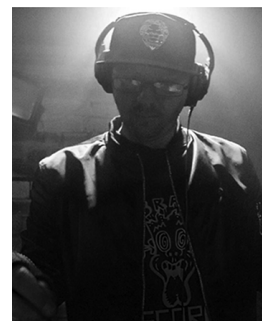

Simon Orchid

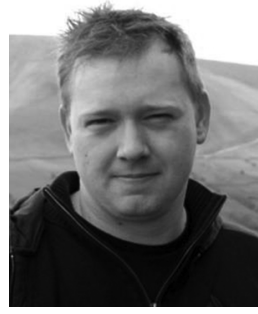

Dr. Whitehead
Daniel Wilson is a Specialist Technician at the University of Manchester, working predominantly in the Laser Processing Research Centre. He is the first point of contact when research students need help and has been named on several research grants with prominent companies. He is working towards completing a part time $\mathrm{PhD}$ in the production of vinyl records using lasers. This combines his day job of designing laser systems with his love of vinyl records. He is currently working on solving the pitfalls with this paper, the focus for the time being is utilizing autofocussing techniques.

Dr. Allegre specializes in advanced laser processes, with a special interest in ultrashort-pulse laser material interactions and process control and a background in precision motion control, optics design, and microscopy. After completing a "diplôme d'Ingénieur" at Polytech' Orleans in France in 2003, he worked as a Design and Software Engineer for research organizations (NCRA, India) and industrial companies (Marinovation, France; Thorlabs, UK). He joined Carl Zeiss SMT Ltd in 2007 as a Systems Engineer working on the design and manufacture of electron microscopes. In 2009, he joined the University of Liverpool to complete a $\mathrm{PhD}$ in advanced control of laser beam wavefront and polarization for micro-manufacturing. In 2012, he managed an EU-funded project to promote the use of laser technologies to SMEs in the North-West.

Robert Heinemann received his Dipl-Ing (FH)in Mechanical Engineering and MSc in Electronic Engineering and Engineering Management from the University of Paderborn at Soest in 1999 and 2001 respectively, and his
$\mathrm{PhD}$ from the University of Manchester Institute of Science and Technology (UMIST) in 2004. Since 2005 he has been working at The University of Manchester, first as a post-doctoral researcher and Leverhulme Trust Early Career Fellow and, since 2013, as a lecturer. His research revolves around machining science, in particular drilling technology as well as process and tool condition monitoring, and the design and development of new tools for minimally invasive surgery.

Simon Orchid is an electronic musician, DJ, vinyl cutting engineer. He studied Creative Music Technology at Bath Spa University. He has over 20 years of experience in various disciplines within music which has been a passion for the majority of his life and has led to work within sound for film, electronics, sound design and has been nominated for a BAFTA for his work with audio for computer games. Simon is currently working as a technician at Wakefield College (UK) alongside pursuing other creative musical interests.

Dr. Whitehead obtained his BSc in Applied Physics in 2001 and then his $\mathrm{PhD}$ in 2005, both at the University of Hull. He specializes in the area of lasers and laser applications. Since 2005, Dr. Whitehead has been working for University of Manchester in the Laser Processing Research Centre (LPRC). In 2009, he was promoted to Senior Experimental Officer, taking over the responsibilities of the laboratory manager for the LPRC. He has overseen the expansion of the LPRC into the Photon Science Institute (PSI) and is currently overseeing the move to new Manchester Engineering Campus Development (MECD). 